\title{
Metaniche session 2016: Tailor-made sensors for rapid Therapeutic Drug Monitoring -Interactions between Biomaterial Physicists, Chemical Engineers and Clinicians for successful translation of technologies in Healthcare
}

\author{
Yoshimi $\mathrm{Y}^{1}$, Pushkala $\mathrm{S}^{2}$, Vijaykumar $\mathrm{H}^{3}$, Dedeepiya VD ${ }^{4}$, Ignacy $\mathrm{A}^{5}$, Swaminathan $\mathrm{J}^{5}$, Rajmohan $\mathrm{M}^{6}$, Karthick R ${ }^{6}, \mathrm{Abraham} \mathrm{SJ}^{4,7}$
}

\begin{abstract}
The Metaniche session is an academic session conducted as a part of Nichi-In Centre for Regenerative Medicine (NCRM)'s novel Initiatives' Conclave in Healthcare Every year (NICHE), the 'NCRM NICHE' organized every year in the month of October by Nichi-In Centre for Regenerative Medicine (NCRM), an IndoJapan academic Institute based at Chennai, India. The Metaniche session aims to bring together the realms of Physics, Chemistry and Biology by portraying inventions or discoveries in physical and chemical sciences which are in the pipeline with high application potential in biology and healthcare. The Metaniche session-2016, held on $22^{\text {nd }}$ October, 2016 in Chennai, India was on the application of biomaterial-based sensors for application in rapid therapeutic drug monitoring (TDM) systems. The session focussed on the role of biomaterials in medicine giving an overview and history of the evolution of biomaterials to suit the growing needs in medicine, followed by emphasizing the need for rapid TDM systems. The final part portrayed the development of tailor-made sensors for rapid TDM using molecularly imprinted polymers as given below.
\end{abstract}

\section{Biomaterials and Medicine}

It has been always medical practitioners coming first to identify the need to lead themselves and others towards the best therapy. When patient services emerged, the clinicians themselves were everybody. Dr Gibbon, when he did his first open heart case in 1950 's, he was the perfusionist and perhaps anaesthetist too. To get into its (bio) materials, through a case study, let us take a review of a simple clinical procedure. Wound closure through suturing. Its tribology matrix highlighted below (Figure 1), sketches its journey, clinical demands, scientific solutions and biomaterials thereof. The bottom line, best is still (re)searched.

Need for rapid therapeutic drug monitoring systems in healthcare

TDM is a most needed much useful tool in the prescription armamentarium of critically ill patients. The good news is that most of the drugs do not need therapeutic monitoring. Drugs that have definable clinical end points such as heart rate, blood pressure, cardiac rhythm, blood sugar, cholesterol and triglycerides need not be monitored. Drugs that have a serum concentration that does not correlate with therapeutic non-toxic effects need not be monitored. Drugs that have pharmacokinetics, which is not a complication and drugs that are not used to treat life threatening diseases need not be monitored. In critically-ill patients antibiotics such as aminoglycosides and vancomycin needs monitoring. Immunosuppressants and anticancer drugs, methotrexate and cyclosporine need monitoring. Antiepileptic drugs, cardiac drugs such as Digoxin, Lidocaine also need to be monitored. Psychoactive drugs such as lithium needs monitoring as well.

The reasons for TDM of these above mentioned drugs are one or more of the following: narrow therapeutic range, poor correlation between dose and serum drug concentration, non-linear pharmacokinetics, good relationship between serum SDC and therapeutic or toxic effects.

The lack of therapeutic effects is dangerous and there is difficulty in interpreting signs and symptoms of toxicity.

The Assay methodologies available for TDM include EMIT, ELISA, RIA, FPIA, HPLC.

The usefulness of TDM is if it is used appropriately it can be used to maximize efficacy and minimize toxicity. It can be useful to monitor levels when hepatic or renal function are challenging and when multiple drugs are used when a drug - drug interaction is anticipated with change of drug levels. Individualizing dosing therapies and making adjustment based on body mass (lean vs obese) and body water (normal vs excessive) can be judiciously made.

Development of tailor-made sensors for therapeutic drug monitoring using molecularly imprinted polymers (MIP)

TDM is the clinical practice of measuring specific drugs at designated intervals to maintain a constant concentration in a patient's bloodstream, thereby optimizing individual dosage regimens. However, conventional analytic methods of drugs require complicated procedure and high cost, and then TDM is 


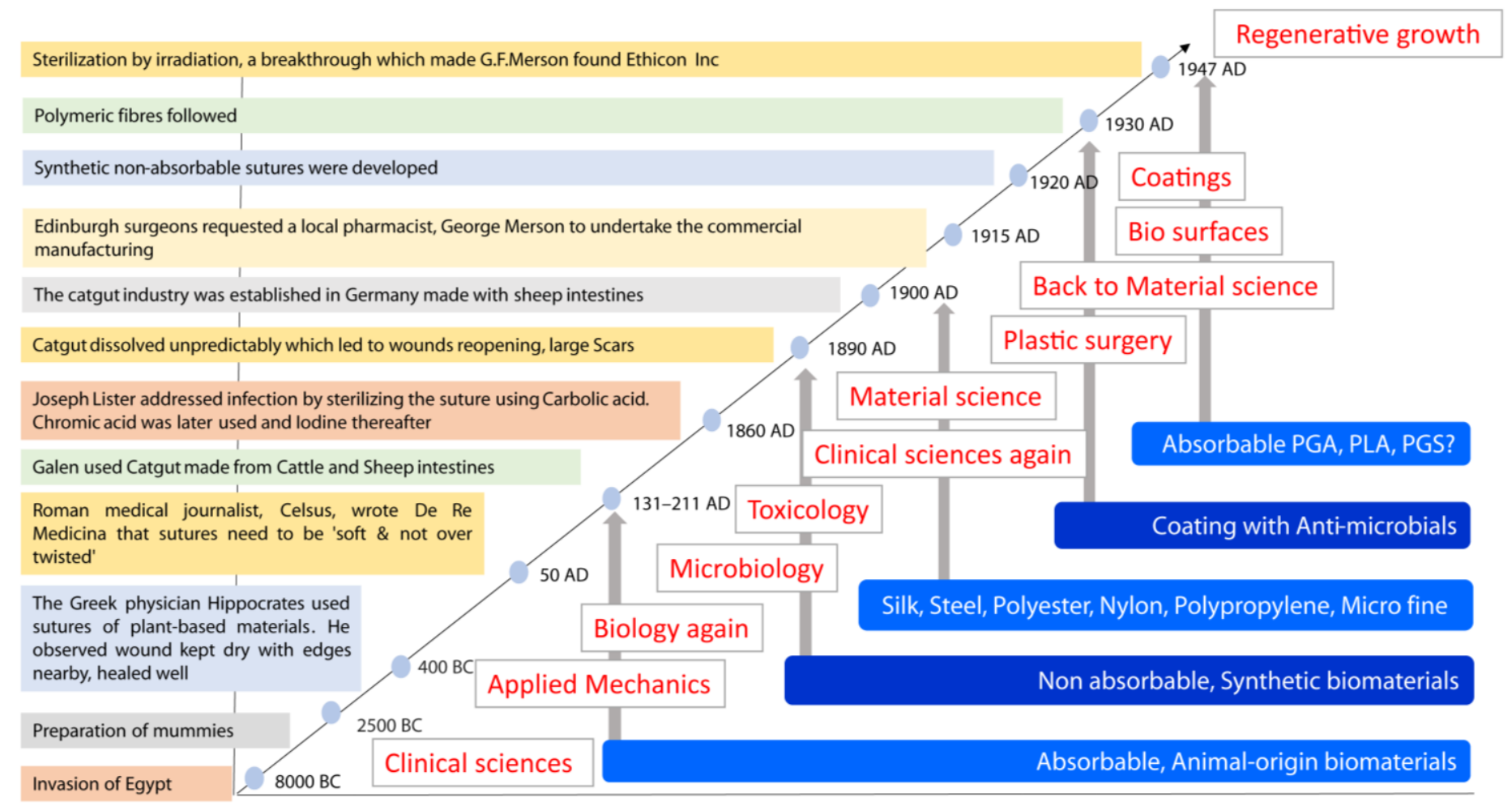

Figure 1: The Transdisciplinarity of biomaterial evolution

Table 1: Assay methodologies available for TDM

\begin{tabular}{|l|l|l|l|}
\hline & Automation & Turnaround & Sensitivity \\
\hline EMIT & Highly & Rapid & Moderate \\
\hline ELISA & Highly & Rapid & Moderate \\
\hline RIA & Trained personnel & Long & High \\
\hline HPLC & Highly trained personnel & Long & Highest \\
\hline
\end{tabular}

hard to perform in small hospitals. Yoshimi's team has been developing sensors using an electrode grafted with thin layer of MIP. The MIP-electrode can detect the template by simple amperometric method, and then it is feasible for drug sensors for TDM.

Outcome of extracorporeal therapy using heart-lung machine depends on appropriate dose of heparin, which is an anticoagulant drug. Blood level of unfractionated heparin is monitored by activated

clotting time (ACT). However, ACT is not sensitive to the level of low molecular weight heparin (LMWH), the utility of which is expanding due to its small side effect. Yoshimi's team has have grafted poly (metacryloxyethyltrimethylamine chloride co- acrylamide -co- methylenebisacrylamide) imprinted with unfractionated heparin on an indium-tin oxide (ITO) electrode. The redox current of ferrocyanide at the grafted electrode can detect LMWH as well as unfractionated heparin in undiluted whole blood. It takes only $30 \mathrm{~s}$ to determine blood level. Then the MIP-electrode would contribute for further expanding utility of $\mathrm{LMWH}^{[1]}$.

Vancomycin is primary drug against infection by gram-positive bacteria. Overdose of vancomycin damages kidney but under dose creates resistant bacteria, then TDM of vancomycin has been recommended strongly. Yoshimi's team have grafted vancomycin-imprinted poly (methacrylic acid -covinylferrocene - $\mathrm{co}$ - acrylamide - $\mathrm{co}$ - methylenebisacrylamide) on ITO electrode. The current detected by differential pulse voltammetry was sensitive to vancomycin concentration over the range $5-60 \mu \mathrm{g} / \mathrm{mL}$ in buffer saline, which covers the clinically required concentration in plasma of $10-40 \mu \mathrm{g} / \mathrm{mL}^{[2]}$. The current was also sensitive to vancomycin concentration in whole undiluted blood over the same range (Figure 2). The method enables reagentless sensing of vancomycin.

Those results demonstrate that MIP-grafted electrodes are feasible for sensors for TDM. 


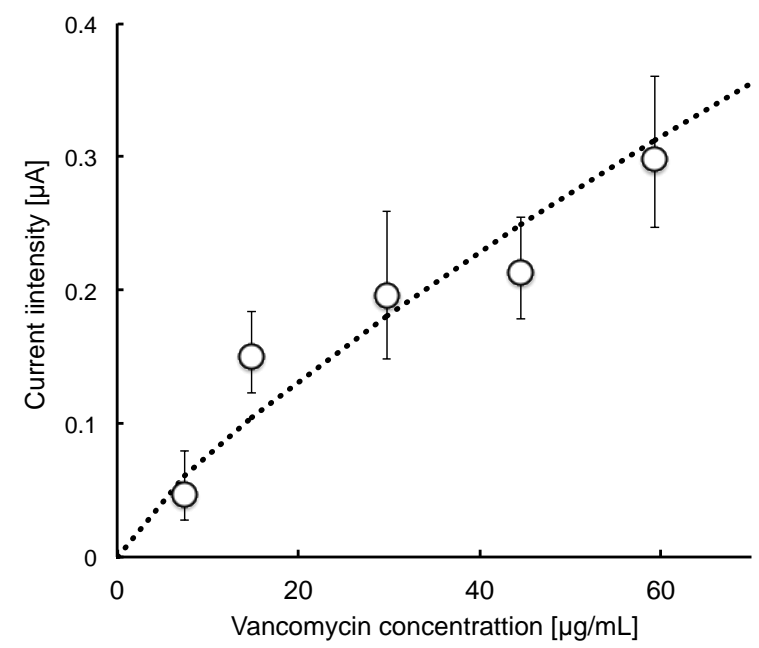

Figure 2: Calibration curve for vancomycin in bovine whole blood $d^{[2]}$

\section{Discussion}

TDM helps to maintain drug concentrations within a targeted therapeutic range or window. Though there are several existing TDM methods and technologies, the hurdles include lack of methods which are fast, more sensitive, portable, reliable, and less invasive. Further, the method should be robust and employ validated bioanalytical assays. Instead of laboratory-based assays, development of bedside assays will reduce resources, time for blood sampling and transporting to laboratories along with increasing the efficiency of the whole process. In this process of developing rapid bedside assays, knowledge, information exchange and interaction between different fields such as physics for identifying the proper raw material, chemistry for analysing and building the ingredients for the assays, biological sciences and medical fields for the application becomes essential. In the current case, use of molecularly imprinted polymers has helped chemical engineers devise a solution for TDM in critical care medicine. The whole process rested on the identification of the need by the clinician followed by interaction with the basic scientists.

Such interactions cannot be possible unless, clinicians and basic science researchers come together in a common platform as suggested by Kong and Segre ${ }^{[3]}$, who have reported that scientists and clinicians often find it difficult to work together and translational research can progress only when there is a proper two way communication and collaboration between basic science researchers and clinicians.

The "Metaniche" session serves this purpose by serving as a platform where clinicians with need get to interact with basic science researchers not only in biological sciences but in the multi-disciplinary fields of physics, chemistry and several others. The "Metaniche" session identifies technologies which are in the pipeline in physics, chemistry or varied other fields and provides an opportunity for the inventors and the biologists/clinicians who will be potential users of the technology thereby fostering collaborations between the two which could lead to improved translation of the technologies in a rapid and a more meaningful manner.

\section{Conclusion}

This year's Metaniche session has represented the needs existing and challenges faced in TDM which has led to the demonstration of how appropriate physical biomaterials and chemical analytical methodologies could come together in creating rapid, bedside TDM sensors, a highly valuable tool in enhancing the lives of critically ill patients. Thus, the "Metaniche" session brings together physics, chemistry and biology, thereby enabling multi-institutional, multidisciplinary interactions to improve the exposure of clinicians to understand and appreciate the potential basic science technologies under development and to enable basic science researchers, to identify, research upon and develop clinically useful knowledge.

\section{References}

1. Yoshimi Y, Inaba R., Ogawa T, Yoshino W, Inoue M, Kuwana K. Stabilized sensing of heparin in whole blood using the 'gate effect' of heparin-imprinted polymer grafted onto an electrode. Molecular Imprinting 2016; 4 (1): 13-20.

2. Yoshimi Y, Ohira H. Development of drug-sensors using MIP-grafted electrode for therapeutic drug monitoring. Proceesings of the 9th International Conference on Molecular Imprinting (MIP2016), 2016; O27, Lund.

3. Kong HH, Segre JA. Bridging the translational research gap: a successful partnership involving a physician and a basic scientist. J Invest Dermatol. 2010 ;130(6):1478-80. 